\title{
Numerical Parameter Identifiability and Estimability: Integrating Identifiability, Estimability, and Optimal Sampling Design
}

\author{
JOHN A. JACQUEZ \\ Department of Physiology, The University of Michigan, Ann Arbor, Michigan 48109-0010 \\ AND \\ PETER GREIF \\ Laboratory of Mathematical Biology, National Cancer Institute, \\ National Institutes of Health, Bethesda, Marvland, 20205
}

Received 20 November 1984; revised 15 May 1985

\begin{abstract}
We define two levels of parameters. The basic parameters are associated with the model and experiment(s). However, the observations define a set of identifiable observational parameters that are functions of the basic parameters. Starting with this formulation, we show that an implicit function approach provides a common basis for examining local identifiability and estimability and gives a lead-in to the problem of optimal sampling design. A least squares approach based on a large but finite set of observations generated at initial parameter estimates then gives a uniform approach to local identifiability, estimability, and the generation of an optimal sampling schedule.
\end{abstract}

\section{INTRODUCTION}

The overall problem of determining the parameter values of a system from input-output data, i.e. the parameter estimation problem, is often called the identification problem. The identifiability problem, on the other hand, is more circumscribed: given a model of the system and specific input-output experiments, we ask, if the data were error free, would the parameters of the model be uniquely determined? The identifiability problem is concerned with the theoretical existence of unique solutions and so is strictly a mathematical and a priori problem. Note that it refers to specific experiments on a specific model, so it is properly model identifiability for the given experiments and not system identifiability. Unfortunately, the term system identifiability is used widely in the literature and really confuses the issue. The identification problem and identifiability have been recognized in a number of fields,

MATHEMATICAL BIOSCIENCES 77:201-227 (1985)

201

OElsevier Science Publishing Co., Inc., 1985

52 Vanderbilt Ave., New York, NY 10017

$0025-5564 / 85 / \$ 03.30$ 
including statistics and econometrics $[1,2]$ and control and systems engineering $[3,4]$, and have received increasing attention in compartmental analysis since the paper by Bellman and Astrom [5], which introduced the term structural identifiability. However, it is important to remember that the two terms are not always used with exactly the same meanings in all of these fields.

Identifiability is such an intriguing problem in basic theory that a large literature has developed on local and global identifiability of linear systems, and now there is a considerable stir about extending this to nonlinear systems. In fact it has received so much attention that we tend to forget at times that it is just one aspect of a larger problem, the inverse problem, which includes identifiability and identification. In a general sense the inverse problcm is the scientist's problem: determine the structure and functioning of a system from experiments and studies of its behavior. The inverse problem encompasses all of the steps involved in determining the structure of a system. It is an iterative process in a number of stages, viz.

(i) Hypothesis generation. This includes specification of one or more models based on the information available at that time.

(ii) Definition of experiments. Not all conceivable experiments can be done, so the choice of experiments is restricted. Availability of resources imposes more constraints.

(iii) Design of experiments. Try to design the experiments so that the model parameters essential for testing the hypotheses can be estimated. This is a large problem that properly includes examination of the effects of varying all facets of the possible experiments that are under the control of the experimenter. Two important steps concern us:

(a) Identifiability and estimability (defined below).

(b) Optimal sampling design.

(iv) Performing the experiments.

(v) Analysis of data and testing of hypothesis.

Identifiability is more than an interesting problem in theory. It is an important step in the inverse process. We are not just interested in whether or not a model is identifiable for a given experiment; even if it is not, we want to know which parameters are identifiable, because we may only need to estimate some of the parameters to test a hypothesis. Furthermore, a set of parameters may be identifiable, but the interactions between the parameters, as measured by correlations for example, may be such as to make numerical estimation of the values of individual parameters difficult. Thus, a parameter can be identifiable but poorly estimable for a given experiment. We want to design the experiment [5] to optimize the estimability of the pertinent 
identifiable parameters. Then we want to choose an optimal sampling design to minimize the errors of estimation. Identifiability, estimability, and optimal sampling design should go together as linked steps in parameter estimation.

Optimal sampling design requires that we have an initial estimate of the parameter values, and in the biological sciences we often do have initial estimates. That suggests a uniform iterative approach: for the possible experiments, test local identifiability at the initial estimates, look at estimability at the initial estimates, and then obtain the optimal sampling design at the initial estimates. Note that we require only local identifiability of particular parameters.

Our long term goal is to develop a practical, uniform method for testing local identifiability and generating an optimal sampling design for the locally identifiable parameters, a method which can be incorporated into a system of modeling such as SAAM or MLAB. In this paper we concentrate on the identifiability problem but propose an integrated approach that includes optimal sampling design. To that end Section II briefly reviews the current state of the art in identifiability. Section III presents the basic theory of local identifiability based on implicit function theory and explores the use of the correlation matrix for the information it contains about local identifiability and estimability at the initial estimates. Section IV then gives a series of examples from compartmental analysis. Section $V$ briefly reviews optimal sampling design and outlines an integrated approach to testing local identifiability and to obtaining an optimal sampling design.

The work in this paper was first conceived in the context of compartmental analysis, and the exposition reflects that influence, but the results are applicable more widely.

\section{REVIEW OF IDENTIFIABILITY}

For linear time invariant models let $\mathbf{x}$ be the vector of state variables, $\mathbf{u}$ a vector of possible inputs, and $\theta$ the vector of model parameters. The equation of the model is

$$
\dot{\mathbf{x}}=\mathbf{A}(\boldsymbol{\theta}) \mathbf{x}+\mathbf{B}(\boldsymbol{\theta}) \mathbf{u}, \quad \mathbf{x}(0)=\mathbf{x}_{0},
$$

in which $\mathbf{A}$ and $\mathbf{B}$ are stationary matrices which are functions of the parameter vector. The vector of observations, $\mathbf{y}$, is given by

$$
\mathbf{y}=\mathbf{C}(\boldsymbol{\theta}) \mathbf{x}+\mathbf{D}(\boldsymbol{\theta}) \mathbf{u}
$$

For compartmental models we usually have $\mathbf{D}(\theta)=\mathbf{0}$ and $\mathbf{B}$ a known matrix not dependent on $\boldsymbol{\theta}$. 
For nonlinear models the corresponding equations are

$$
\begin{aligned}
& \dot{\mathbf{x}}=\mathbf{f}(\mathbf{x}, \mathbf{B u}, \boldsymbol{\theta}, t), \quad \mathbf{x}(0)=\mathbf{x}_{0}, \\
& \mathbf{y}=\mathbf{G}(\mathbf{x}, \mathbf{B u}, \boldsymbol{\theta}, t)
\end{aligned}
$$

Note that $\mathbf{A}(\boldsymbol{\theta})$ and $\mathbf{f}$ specify the model, Bu specifies the input for a particular experiment and $\mathbf{y}$ the observations. An experiment is specified by the initial conditions $\mathbf{x}(0)$, the inputs $\mathbf{B u}$ from among some set of possible inputs $\mathbf{u}$, and the observations $\mathbf{y}$.

Let $\hat{\theta}$ be value of $\theta$ that gives the response of a model to a given experiment, i.e. a solution of Equation (2) or (4). A parameter vector is (structurally) locally identifiable if for almost any solution $\hat{\theta}$ the solution is unique in some neighborhood of $\hat{\boldsymbol{\theta}}$. The term "almost any solution" means except for possibly a set of zero measure. This caveat is included because there may be some special combinations of parameter values for which a unique solution cannot be found. The definition of (structural) global identifiability is the same except that the solution must then be unique for the entire domain of $\boldsymbol{\theta}$, not just a neighborhood of $\boldsymbol{\theta}$.

Linear identifiability has been reviewed by Nguyen and Wood [6]. For a more general review see the monograph of Walter [7], and for compartmental systems see Anderson [8] and the brief review by Jacquez [9]. For more detail References [10-27] are recommended. A detailed review and discussion of the different ideas and terminologies in this field will be found in the recent thesis of Delforge [28].

To put the remainder of this paper in context we review briefly the major approaches that have been used to check identifiability for linear time invariant compartmental systems. The equations for such a system are

$$
\begin{aligned}
& \dot{\mathbf{x}}=\mathbf{A x}+\mathbf{B u}, \quad \mathbf{x}(0)=\mathbf{x}_{0}, \\
& \mathbf{y}=\mathbf{C x}
\end{aligned}
$$

In general $\mathbf{B}$ is a matrix of known elements; $\mathbf{C}$ is often known, but may include unknown elements, i.e. parameters. At the simplest level $\mathbf{B}$ and $\mathbf{C}$ are known, so the identifiability problem reduces to whether the nonzero elements of $\mathbf{A}$ are uniquely specified by the observations $\mathbf{y}$ for the given inputs Bu. The following methods handle unknown parameters in $\mathbf{B}$ and $\mathbf{C}$ as well.

\section{A. THE TRANSFER FUNCTION OR IMPULSE RESPONSE METHOD}

Consider the experiment given by Equations (5) and (6) but with a zero initial state. If the impulse response were determinable without error, that would specify the transfer function. Take Laplace transforms of (5) and (6) 
and solve for the Laplace transform $\mathbf{Y}$, of the observation $\mathbf{y}$ :

$$
\mathbf{Y}=\mathbf{C}(s \mathbf{I}-\mathbf{A})^{-1} \mathbf{B U} \text {. }
$$

Let us call $\mathbf{H}(s)=(s \mathbf{I}-\mathbf{A})^{-1}$ the transfer function for the model, because $H_{i j}(s)$ gives the observation in compartment $i$ for unit impulse into $j$. Then $\mathbf{C}(s \mathbf{I}-\mathbf{A})^{-1} \mathbf{B}$ is the transfer function for the experiment, because it specifies what combinations of the $H_{i j}(s)$ are available from the experiment with input $\mathbf{B u}$ and observation y. We assume the transfer functions are written in a canonial form, i.e., common factors in numerator and denominator are canceled and the transfer function is simplified so that the coefficient of the highest power of $s$ in the denominator is always 1 . The problem then reduces to an examination of the coefficients of the terms in the transfer function for the experiment to determine whether all of the nonzero components of $\mathbf{A}$ are uniquely specified, and this is a problem in solving simultaneous nonlinear equations.

Closely related to the transfer function approach is the examination of the Markov parameters [29], which is also used in the testing of controllability and observability in control system theory.

Anderson [8] has taken initial steps toward solving the simultaneous polynomial equations obtained from the transfer function by making use of the particular structure of these equations. Also see the paper by Raksanyi et al. [30] in this issue on solving simultaneous polynomial equations obtained from the transfer function and other approaches.

\section{B. THE SIMILARITY TRANSFORMATION METHOD}

Another approach is to seek all models that have matrices related to $A$ by a similarity transformation and that also satisfy all constraints on the model and give the same input-output response for the experiment. The first attack on the identifiability problem for compartmental models was by Berman and Schoenfeld [31], who used the similarity transformation approach to generate the set of output-indistinguishable models. The more recent definitive work is due to Travis and Haddock [24], Walter and Lecourtier [27], and Vajda [26].

Let $\mathbf{P}$ be nonsingular and replace $\mathbf{A}$ by $\mathbf{P}^{-1} \mathbf{A P}$ in Equation (5). Taking Laplace transforms, again for $\mathbf{x}(0)=\mathbf{0}$, gives

$$
\mathbf{Y}=\mathbf{C P}^{-1}(s \mathbf{I}-\mathbf{A})^{-1} \mathbf{P B U} .
$$

Thus $\mathbf{P}^{-1} \mathbf{A P}$ must satisfy all of the constraints on the model, and for the input-output response to remain unchanged, $\mathbf{C P}^{-1}=\mathbf{C}$ and $\mathbf{P B}=\mathbf{B}$. If on applying these conditions to determine the elements of $\mathbf{P}$, we find $\mathbf{P}=\mathbf{I}$, then the model is uniquely identifiable. The same results are obtained by taking $\mathbf{P}$ as a nonsingular transformation on $\mathbf{x}, \mathbf{x}^{\prime}=\mathbf{P x}$. 


\section{THE MODAL MATRIX AND EIGENVALUES}

Assume the eigenvalues are distinct, a not unreasonable assumption in many applications. There is a map from the $n^{2}$ elements of $\mathbf{A}$ to the $n^{2}$ independent components of the eigenvectors and eigenvalues, so instead of trying to identify the elements of $\mathbf{A}$ one might try to identify the eigenvalues and the matrix of eigenvectors, the modal matrix $[17,18,22,23]$.

Let $\mathbf{M}$ be the modal matrix, and $\Lambda$ be the diagonal matrix of eigenvalues. Then $\mathbf{A}=\mathbf{M} \Lambda \mathbf{M}^{-1}$, and the impulse response can be written

$$
\mathbf{y}=\mathbf{C} e^{\mathbf{M} \Lambda M^{-1} t} \mathbf{B}=\mathbf{C M} e^{\Lambda t} \mathbf{M}^{-1} \mathbf{B}
$$

Let $\mathbf{r}_{i}^{T}$ be the $i$ th row of $\mathbf{M}$, and $\mathbf{c}_{j}$ the $j$ th column of $\mathbf{M}^{1}$. The relations between elements of $\mathbf{A}$ and $\mathbf{M}$ are

$$
\begin{aligned}
A_{i j} & =\mathbf{r}_{i}^{T} \Lambda \mathbf{c}_{j}, \\
A_{0 j} & =-\sum \mathbf{r}_{i}^{T} \Lambda \mathbf{c}_{j}, \\
\mathbf{r}_{i}^{T} \mathbf{c}_{j} & =\delta_{i j}
\end{aligned}
$$

For zero initial state the impulse response of compartment $i$ for unit input into $j$ at $t=0$ is given by

$$
x_{i}=\mathbf{r}_{i}^{T} e^{\Lambda /} \mathbf{c}_{j}
$$

The problem is to use all available observations plus any known constraints on the $A_{i j}$ to see if the elements of $\mathbf{M}$ and $\Lambda$ are uniquely specified.

Another method examines the coefficients of the Taylor series expansion of the observations to see if all parameters are uniquely identifiable [25].

All of these methods work well with small compartmental systems but become increasingly difficult to apply as the system increases in size. Such limitations, taken in conjunction with the need for practical methods, provides another argument for emphasizing local identifiability for particular significant parameters rather than global and model identifiability.

\section{BASIC THEORY FOR LOCAL IDENTIFIABILITY}

We develop a general approach to local identifiability which makes use of linearization in parameter space around the initial estimates and application of implicit function theory. Grewal and Glover [12] have shown that local identifiability of the linearized form of a nonlinear model implies local identifiability of the nonlinear model. It is possible, however, for the nonlinear model to be locally identifiable and for the linearized form not to be. The development starts from the recognition that there is a common general 
structure to the identifiability problem, that in applications we are primarily interested in identifiability for the parameters falling in certain ranges of values, and that in fact we often have initial estimates for the values of the parameters.

\section{A. GENERAL STRUCTURE OF THE IDENTIFIABILITY PROBLEM}

Choosing a model specifies the state variables $(\mathbf{x})$ and the parameters of the model $\left(\theta^{*}\right.$, a $p^{*}$-vector). An experiment specifies the initial state, the input $\mathbf{B u}$ from some vector of inputs $\mathbf{u}$ and the observations. The model response to input Bu for the initial conditions is the solution of the structural equation (14) for the initial conditions

$$
\mathbf{F}\left(\mathbf{x}, \dot{\mathbf{x}}, \ldots, \boldsymbol{\theta}^{*}, \mathbf{B u}, t\right)=\mathbf{0}, \quad \mathbf{x}(0)=\mathbf{x}_{0} .
$$

F may be a function of derivatives but need not necessarily be so. It should be noted that for some inputs only a subset of the parameters may actually appear in the model response. The observations are given by some function of the state variables, the input $\mathbf{B u}$, and a vector of parameters, $(\varphi$, an $m$-vector):

$$
\mathbf{y}=\mathbf{G}(\mathbf{x}, \boldsymbol{\varphi}, \mathbf{B u}, t)
$$

We distinguish between three types of parameters, structural, experimen$t a l$, and observational. The structural parameters are inherent to the model and so appear in the structural equation (14). But, and we repeat for emphasis, for some inputs it is possible that only a subset of the parameters in $\theta^{*}$ actually appear in the solution of the structural equations for that input. Appropriate choice of $\mathbf{B u}$ is needed to make sure all structural parameters appear in the solutions of Equation (14); this is the condition of input reachability $[15,32]$ for compartmental models.

The experiment specifies initial conditions [the input $\mathbf{B u}$ ] and the observations [Equation (15)], and may introduce parameters by way of each of these. We call such parameters experimental parameters and denote them by $\theta^{\prime}$, a vector of $p^{\prime}$ components. These are at the same level as the structural parameters, so the basic parameter vector is $\theta=\left[\begin{array}{ll}\theta^{* T} & \theta^{\prime T}\end{array}\right]^{T}$ of order $p=p^{*}+$ $p^{\prime}$. The initial conditions are often fully specified in biological experiments, and we shall not here consider cases in which experimental parameters are introduced through the initial conditions. But, as one of the referees has pointed out, even when specified, some initial conditions may be parameters that need to be measured, and just as it is possible for some structural parameters not to influence the observations, some of the initial conditions may not influence the observations. The inputs Bu may also introduce experimental parameters, but that is not often true in biological experimenta- 
tion, so we pay little attention to it. Note that the structural parameters plus those experimental parameters introduced through initial conditions and inputs have the potential to appear in the solutions to the structural equations for the given initial conditions and inputs. The other experimental parameters, those introduced by the process of observation, cannot appear in the solution to the structural equations, but must appear in the equations for the observations (15); such experimental parameters are not uncommon in biology. If we could be assured that the initial conditions are fully known, as in some experiments with radioactive tracers, then we could think of the experimental parameters as those introduced by way of the inputs and the observations.

Finally there is the vector of observational parameters, $\varphi$, a vector of $m$ components. These are, by definition, the uniquely determinable parameters obtainable from the equations of the observations; the observational parameters are functions of the structural and experimental parameters that appear in the observations. Thus $\Phi$ is at a level different from $\theta$. All of the components of $\theta^{*}$ may not appear in $\varphi$, so $\varphi$ is generally a function of $\theta^{\prime}$ and of those components of $\theta^{*}$ that are passed on to the observations; in general $m \leqslant p$, and often $m<p$. Note that the $\varphi$ are not necessarily unique in functional form; their functional form depends on the approach used to examine the observations (i.e. transfer function or other), but all approaches must generate uniquely determinable sets of observational parameters that contain the same information about $\theta$. Thus, the structural invariants of Vajda [26] and the exhaustive summary of Walter [7] are observational parameters.

Now we can reformulate the identifiability problem: Are the structural and experimental parameters uniquely determined by the observational parameters? If the experiment introduces no new parameters, we have the identifiability problem in its simplest and cleanest form: Do the observational parameters uniquely determine the structural parameters? Note that only certain components of $\boldsymbol{\theta}$ may need to be identifiable to test a particular hypothesis; then we need only test whether the relevant structural and experimental parameters are uniquely determined by the observational parameters.

Finally, we emphasize that the method we develop makes use of the idea of observational parameters in the derivation, but it is not necessary to generate a specific set $\boldsymbol{\varphi}$ in order to determine whether particular components of $\boldsymbol{\theta}$ are locally identifiable.

\section{B. THEORY OF OBSERVATIONS IF Y IS A SCALAR}

We develop the basic theory assuming $y$ to be a scalar; the extension to a vector of observations is straightforward and adds only algebraic complexity. For local identifiability the theory can be developed as an integral theory or 
based on a finite number of samples; the latter path is taken here for uniformity with the optimal sampling theory.

In optimal sampling theory (Section $V$ ) we assume a finite number of samples, $n$, with measurement error $\epsilon_{i}$ added to the observation $y_{i}$, as in

$$
\eta_{i}=y_{i}+\epsilon_{i}=G\left(\mathbf{x}_{i}, \boldsymbol{\varphi}, \mathbf{B u}, t_{i}\right)+\epsilon_{i} .
$$

We then linearize the observations around initial estimates $\theta^{0}$ of the parameters. These initial estimates are "true values" for the simulated data generated from a model. A least squares approach is then used to find optimal sampling times.

We want to follow a similar path to check local identifiability of the parameters at their initial estimates $\boldsymbol{\theta}^{0}$. But identifiability is an a priori problem; there are no measurement errors. Examine small deviations in $\boldsymbol{\theta}$ around $\theta^{0}$ by expanding $y_{i}$ in Taylor series:

$$
y_{i}=G\left(\mathbf{x}_{i}, \varphi^{0}, \mathbf{B u}, t_{i}\right)+\sum_{j=1}^{p} \frac{\partial G_{i}^{0}}{\partial \theta_{j}} \Delta \theta_{j}+e_{i} .
$$

Here $\partial G_{i}^{0} / \partial \theta_{j}$ is the derivative evaluated at $\theta^{0}$, and $e_{i}$ is the contribution of higher order terms, the error of approximation by linearization. Form the sum of squared deviations for the linearized form, equation (18).

$$
S=\sum_{i=1}^{n}\left[y_{i}-G_{i}^{0}-\sum_{j=1}^{p} \frac{\partial G_{i}^{0}}{\partial \theta_{j}} \Delta \theta_{j}\right]^{2} .
$$

$S$ is simply the square of the sum of the higher order terms in the Taylor expansion, so $S$ is zero at $\theta=\theta^{0}$. Note that $n$ is some large number of points at which we generate observations from the model.

Taking derivatives with respect to $\Delta \theta_{k}$ gives the normal equations for the estimates $\widehat{\Delta \boldsymbol{\theta}}_{k}$ :

$$
\sum_{j=1}^{p} \sum_{i=1}^{n}\left(\frac{\partial G_{i}^{0}}{\partial \theta_{j}} \frac{\partial G_{i}^{0}}{\partial \theta_{k}}\right) \widehat{\Delta \theta}_{j}=\sum_{i=1}^{n}\left(y_{i}-G_{i}^{0}\right) \frac{\partial G_{i}^{0}}{\partial \theta_{k}}, \quad k=1, \ldots, p .
$$

Let $z_{i}=y_{i}-G_{i}^{0}$, so $\mathbf{z}=\left(z_{1} \cdots z_{n}\right)^{T}, \widehat{\Delta \theta}=\left(\widehat{\Delta \theta}_{1}, \ldots, \widehat{\Delta \theta}_{p}\right)^{T}$. Define the matrix $\mathbf{g}$ by

$$
\mathbf{g}=\left[\begin{array}{ccc}
\frac{\partial G_{1}^{0}}{\partial \theta_{1}} & \cdots & \frac{\partial G_{1}^{0}}{\partial \theta_{p}} \\
\vdots & & \vdots \\
\frac{\partial G_{n}^{0}}{\partial \theta_{1}} & \cdots & \frac{\partial G_{n}^{0}}{\partial \theta_{p}}
\end{array}\right] .
$$


The sum of squares and normal equations are given by

$$
\begin{aligned}
S & =(\mathbf{z}-\mathbf{g} \Delta \boldsymbol{\theta})^{T}(\mathbf{z}-\mathbf{g} \Delta \boldsymbol{\theta}), \\
\mathbf{g}^{T} \mathbf{g} \widehat{\Delta \boldsymbol{\theta}} & =\mathbf{g}^{T} \mathbf{z} .
\end{aligned}
$$

Now note that we can repeat this derivation in terms of the observational parameters to obtain

$$
\mathbf{g}_{\varphi}^{T} \mathbf{g}_{\varphi} \widehat{\Delta \varphi}=\mathbf{g}_{\varphi}^{T} \mathbf{z}
$$

$\mathbf{g}_{\Psi}$ is given by

$$
\mathbf{g}_{\varphi}=\left[\begin{array}{ccc}
\frac{\partial G_{1}^{0}}{\partial \varphi_{1}} & \cdots & \frac{\partial G_{1}^{0}}{\partial \varphi_{m}} \\
\vdots & & \vdots \\
\frac{\partial G_{n}^{0}}{\partial \varphi_{1}} & \cdots & \frac{\partial G_{n}^{0}}{\partial \varphi_{m}}
\end{array}\right]
$$

Let $\mathbf{J}$ be the Jacobian of $\varphi$ with respect to $\boldsymbol{\theta}$,

$$
\mathbf{J}=\left[\begin{array}{ccc}
\frac{\partial \varphi_{1}}{\partial \theta_{1}} & \cdots & \frac{\partial \varphi_{1}}{\partial \theta_{p}} \\
\vdots & & \vdots \\
\frac{\partial \varphi_{m}}{\partial \theta_{1}} & \cdots & \frac{\partial \varphi_{m}}{\partial \theta_{p}}
\end{array}\right] .
$$

Then $\mathbf{g}=\mathbf{g}_{\varphi} \mathbf{J}$ and $\mathbf{g}^{T} \mathbf{g}=\mathbf{J}^{T} \mathbf{g}_{\varphi}^{T} \mathbf{g}_{\varphi} \mathbf{J}$.

In anticipation of the review of optimal sampling theory and the sequel, Section V, it is worthwhile emphasizing the uniformity of basic theory in that both local identifiability and optimal sampling theory are developed with a least squares approach. For local identifiability the errors of observation are zero, so $z_{i}=y_{i}-G_{i}^{0}$ is simply the deviation of $y_{i}$ from its value at $\theta^{0}$. For the optimal sampling derivation, random errors of known variance are added to the calculated values.

\section{IMPLICATIONS FOR LOCAL IDENTIFIABILITY}

In all that follows we assume that enough samples have been generated so that $n>p, n>m$.

By assumption the $\varphi_{i}$ are linearly independent, so the rank of $\mathbf{g}_{\varphi}^{T} \mathbf{g}_{\varphi}$ is $m$ and $\mathbf{g}_{\varphi}^{T} \mathbf{g}_{\varphi}$ has an inverse. The identifiability problem is to examine $\mathbf{g}^{T} \mathbf{g}$ to see if the components of $\boldsymbol{\theta}$ are identifiable, so we examine the rank of $\mathbf{g}^{T} \mathbf{g}$ and its relation to $\mathbf{g}_{\varphi}^{T} \mathbf{g}_{\varphi}$. The rank of $\mathbf{g}^{T} \mathbf{g}$ must be $m$. 
1. Rank of $\mathbf{g}^{T} \mathbf{g}$ and Model Identifiability. Now we examine as series of generic situations that occur in compartmental systems analysis to see what they imply for the rank and inverse of $\mathbf{g}^{T} \mathbf{g}$.

(i) One of the structural parameters does not appear in the observations. Assume it is $\theta_{j}$. Then $\partial G_{i}^{0} / \partial \theta_{j}=0$ for $i=1, \ldots, n$. In consequence column $j$ of $\mathbf{g}$ is null, so row $j$ and column $j$ of $\mathbf{g}^{T} \mathbf{g}$ are null and $\left|\mathbf{g}^{T} \mathbf{g}\right|=0$. Note that if $\theta_{j}$ does not appear in the observations, none of the observational parameters are functions of $\theta_{j}$, so $\partial \varphi_{i} / \partial \theta_{j}=0, i=1, \ldots, m$. Then column $j$ of $\mathbf{J}$ is null, and so is column $j$ of $\mathbf{g}=\mathbf{g}_{q} \mathbf{J}$. For what sort of situations do we find that a component $\theta_{j}$ does not appear in the observations? In compartmental models there are two types of experiments in which this occurs. The first is when the model is not input reachable, so that some compartments are not influenced by the input. In that case, fractional transfer coefficients for paths that can be traversed only from compartments that are not input reachable to observed compartments do not appear in the observations; such parameters do not even appear in the model response, i.e. the solution to (14). The other situation occurs when the model is not output reachable. In that case there are transfers from some compartments that do not appear in the set of all possible paths to the observed compartments. The fractional transfer coefficients for those transfers may appear in the model response equations, but not in the observations [Equation (15)].

(ii) All basic parameters $\theta$ appear in the observations. If $m<p,\left|\mathbf{g}^{T} \mathbf{g}\right|=0$. Since $\mathbf{g}^{T} \mathbf{g}=\mathbf{J}^{T} \mathbf{g}_{\varphi}^{T} \mathbf{g}_{\varphi} \mathbf{J}$, we have that $\mathbf{g}^{T} \mathbf{g}$ is a product of rectangular matrices; and for a product $\mathbf{A B}$, if $\mathbf{A}$ is $n \times m, \mathbf{B}$ is $m \times n$, and $m<n$, then $|\mathbf{A B}|=0$ [33]. If $m=p$ then $\mathbf{J}$ is $m \times m$, and since the rank of $\mathbf{g}_{\varphi}$ is $m$, the rank of $\mathbf{g}^{T} \mathbf{g}$ is determined by the rank of $\mathbf{J}$. If $\left|\mathbf{J}^{T} \mathbf{J}\right| \neq 0$, the model is locally identifiable; but $\left|\mathbf{J}^{T} \mathbf{J}\right| \neq 0$ is a sufficient not necessary condition for local identifiability [28].

In both situations (i) and (ii), if $\left|\mathbf{g}^{T} \mathbf{g}\right|=0$ then $\left|\mathbf{J}^{T} \mathbf{J}\right|=0$. But, as we will see in Section III.C.3, we need not generate $\mathbf{J}$ explicitly to test for local identifiability.

2. Parameter Identifiability and Structure of $\boldsymbol{g}^{T} \boldsymbol{g}$. Rank conditions tell us about model identifiability, but in applications it may be more important to find out which parameters are identifiable. That information lies in the relations between the $\varphi$ and $\theta$. The important question is whether one can get at it even if one does not know what the exact functional relations are between the components of $\varphi$ and of $\theta$. To that end we first look at the structure of $\mathbf{g}^{T} \mathbf{g}$. For other approaches see Walter [7, Chapter 3] and Milanese and Sorrentino [34].

To see what the implications for the structure of $\mathbf{g}^{T} \mathbf{g}$ are, we classify the parameters $\theta$ into three groups. To simplify matters we number the components of $\boldsymbol{\theta}$ in a particular order, so the structure of $\mathbf{g}^{T} \mathbf{g}$ obtained results from 
row and column interchanges if the components were not originally numbered in that order:

(i) Locally identifiable. Let $\theta_{1}, \ldots, \theta_{u}$ be the parameters that are locally identifiable. There are $u$ relations of the form

$$
\begin{gathered}
\varphi_{1}=\varphi_{1}\left(\theta_{1}, \ldots, \theta_{u}\right), \\
\vdots \\
\varphi_{u}=\varphi_{u}\left(\theta_{1}, \ldots, \theta_{u}\right),
\end{gathered}
$$

for which the Jacobian $\mathbf{J}_{u}$ generally has nonzero determinant, $\left|\mathbf{J}_{u}\right| \neq 0$. Note that it is possible for $\left|\mathbf{J}_{u}\right|=0$, but that occurs on a set of measure zero and so will not hold for almost all nearby points in the parameter space.

(ii) Locally nonidentifiable but observable. If a parameter is transmitted into the observations, we say the parameter is observable. Note this is different from Kalman's [35] observability of the state vector. Let $\theta_{u+1}, \ldots, \theta_{v}$ be the parameters that are not locally identifiable but which do appear in the observations:

$$
\begin{gathered}
\varphi_{u+1}=\varphi_{u+1}\left(\theta_{1}, \ldots, \theta_{u}, \theta_{u+1}, \ldots, \theta_{v}\right), \\
\vdots \\
\varphi_{m}=\varphi_{m}\left(\theta_{1}, \ldots, \theta_{u}, \theta_{u+1}, \ldots, \theta_{v}\right) .
\end{gathered}
$$

The Jacobian, $\mathbf{J}_{v}$, is of rank $m-u$.

(iii) Nonobservable and nonidentifiable. Finally we have a subset, $\theta_{v+1}, \ldots, \theta_{p}$, of parameters which do not even appear in the observations. They cannot be identifiable. With this partition of the parameters $\theta_{1}, \ldots, \theta_{p}$, $\mathbf{J}$ must have the structure

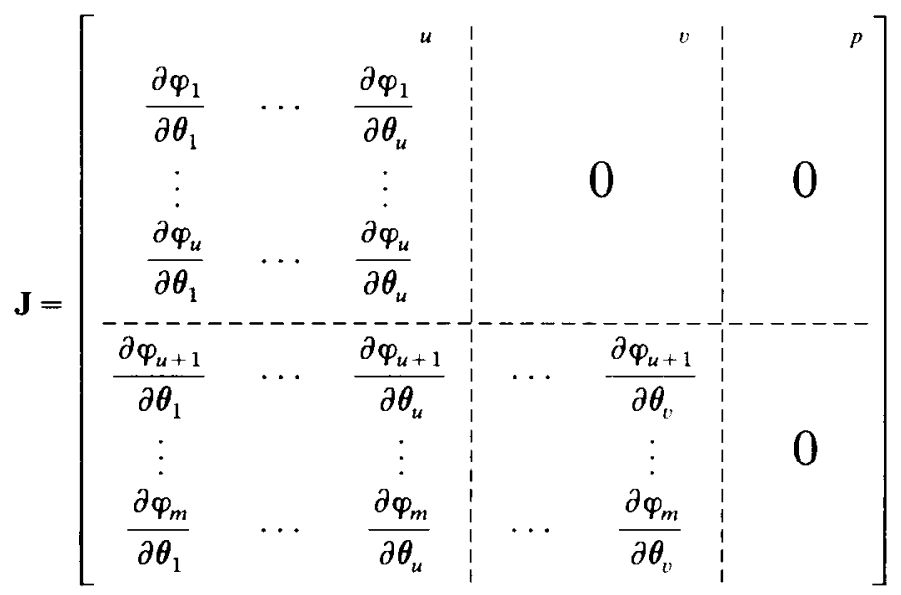

$$
\begin{aligned}
& =\left[\begin{array}{lll}
\mathbf{J}_{u} & \mathbf{0} & \mathbf{0} \\
\mathbf{X} & \mathbf{V} & \mathbf{0}
\end{array}\right] \text {. }
\end{aligned}
$$


Partition $\mathbf{g}_{p}$ conformably as

$$
\mathbf{g}_{\boldsymbol{\varphi}}={ }_{m}^{u}\left[\begin{array}{c:c}
{ }_{m} & \mathbf{B}^{m} \\
\hdashline \mathbf{A} & \mathbf{B} \\
\hdashline \mathbf{D}
\end{array}\right]
$$

Thus

$$
\mathbf{g}_{\varphi} \mathbf{J}=\left[\begin{array}{c:c:c}
\mathbf{A} \mathbf{J}_{u}+\mathbf{B x} & \mathbf{B V} & \mathbf{0} \\
\hdashline \mathbf{C J _ { u }}+\mathbf{D X} & \mathbf{D V} & \mathbf{0}
\end{array}\right],
$$

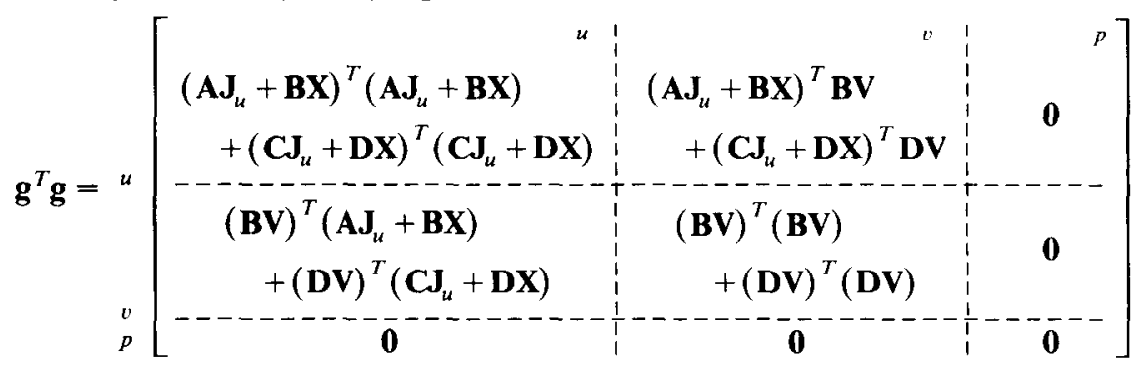

3. The Parameter Correlation Matrix and Parameter Identifiability. The structure of $\mathbf{g}^{T} \mathbf{g}$ clearly points to the parameters which do not appear in the observations. But the identification of all three types of parameters is not so obvious. Provided we are not at one of the points where $\left|J_{u}\right|=0$, the parameter correlation matrix provides considerable information to separate the three groups. To show this we start with Equation (23), which has for its solution

$$
\widehat{\Delta \varphi}=\left(\mathbf{g}_{\varphi}^{T} \mathbf{g}_{\varphi}\right)^{-1} \mathbf{g}_{\varphi}^{T} \mathbf{z}
$$

But $\mathbf{z}=\mathbf{0}$, so the solution is $\widehat{\Delta \varphi}=\mathbf{0}$, as expected. The equations for the first group of parameters are

$$
J_{u} \widehat{\Delta \theta}_{u}=\widehat{\Delta \varphi}_{u}=\mathbf{0},
$$

in which $\widehat{\Delta \theta}_{u}$ and $\widehat{\Delta \Phi}_{u}$ are the restrictions of the parameters to the first $u$ components. Herc $\widehat{\Delta \theta}_{u}=\mathbf{0}$ because $\left|\mathbf{J}_{u}\right| \neq 0$.

Now consider the equations for set (ii):

$$
\begin{gathered}
\frac{\partial \varphi_{u+1}}{\partial \theta_{u+1}} \widehat{\Delta \theta}_{u+1}+\cdots+\frac{\partial \varphi_{u+1}}{\partial \theta_{v}} \widehat{\Delta \theta}_{v}=0, \\
\vdots \\
\frac{\partial \varphi_{m}}{\partial \theta_{u+1}} \widehat{\Delta \theta}_{u+1}+\cdots+\frac{\partial \varphi_{m}}{\partial \theta_{v}} \widehat{\Delta \theta}_{v}=0 .
\end{gathered}
$$


We have already shown that $\widehat{\Delta \theta}_{i}=0$ for $i=1, \ldots, u$, so the terms corresponding to these have been dropped from (34). There are more unknowns than equations in (34), so this is an underdetermined set of equations.

Finally, $\theta_{v}, 1, \ldots, \theta_{P}$ are completely independent of the other parameters; they do not appear in the observations.

Now examine the correlations between the parameters. The correlation matrix has the following structure for off-diagonal elements; the diagonal elements are always 1.

(1) Locally identifiable parameters will have correlations with all parameters $-1<r_{i}<1$, but usually nonzero because they are generally not orthogonal.

(2) For all parameters in set (ii), each parameter has a correlation of +1 or of -1 with at least one other parameter of the set. Because the equations (34) are functionally underdetermined, a small change in a parameter can always be compensated by another change in at least one other in the set. This gives a pattern of correlations of +1 and -1 to define set (ii). The correlations between a member of set 1 and one of set (ii) fall between +1 and -1 . Note that parameters in set (ii) are locally unidentifiable for the linearized equations (34). It is possible for a locally identifiable parameter to appear in this set; the local identifiability can then only be determined from examination of higher order terms in the expansion of $\varphi$ [28].

(3) Since the observations are independent of the parameters in set (iii), the correlation between members of set (iii) and all of the remaining parameters must be zero.

Remark. In applications it is possible to generate sample sets that carry information primarily on combinations of locally identifiable parameters, and as a result of roundoff come up with apparent correlations of \pm 1 . Checks of other parameter ranges then give correlations that are not \pm 1 . For nonidentifiable parameters a correlation of \pm 1 does not change as one changes parameter values.

\section{EXAMPLES}

In this section we present a few examples to show how the methods developed in the previous section work on problems involving linear compartmental models. We present the model and a number of possible experiments. For each experiment we tell whether the model is input reachable and output reachable [32], and exhibit the elements of $\varphi$ as defined by the coefficients in the transfer function for the experiment. Then we give the results of a simulation study for a given set of parameter $\left(\theta^{0}\right)$ values, the rank of $\mathbf{g}^{T} \mathbf{g}$, and finally the correlation matrix, which was calculated with the use of the modeling software package SAAM. 


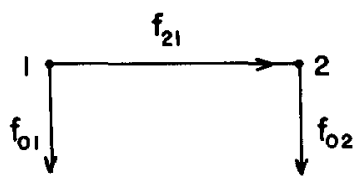

FIG. 1.

\section{A. EXAMPLE 1}

For the simplest example we use the model shown in Figure 1 for impulsive inputs into compartment 1.

Experiment 1a: Input into 1, Observe 1.

Input reachable (IR): yes.

Output reachable (OR): no.

Transfer function: $Q_{1}=1 /\left(s+f_{01}+f_{21}\right)$.

$\varphi_{1}=f_{01}+f_{21} ; f_{02}$ does not appear in the observations.

Simulation: $f_{21}=1.0, f_{02}=2.0, f_{01}=0.5$.

$G(\mathbf{x}, \boldsymbol{\theta}, \mathbf{B u}, t)$ was calculated at 50 uniformly spaced points over the interval $[0,1]$.

$\mathbf{g}^{T} \mathbf{g}$ is of rank $1 ;\left|\mathbf{g}^{T} \mathbf{g}\right|=0$.

The correlation matrix obtained is

$$
\mathbf{C M}=\left[\begin{array}{rrr}
f_{21} & f_{02} & f_{01} \\
& 0 & -1 \\
& 1 & 0 \\
& & 1
\end{array}\right]
$$

Note that $f_{02}=\theta_{2}$ is not observable and therefore not identifiable, and the correlation coefficients $r_{12}, r_{23}$ are zero. Furthermore $\varphi_{1}=f_{21}+f_{01}$ is identifiable, but $f_{21}$ and $f_{01}$ are not individually identifiable, and as expected, $r_{13}=-1$.

Experiment Ib: Input into 1, Observe 2.

IR: yes.

OR: yes.

The transfer function for the observations is

$$
Q_{2}=\frac{f_{21}}{s^{2}+\left(f_{01}+f_{02}+f_{21}\right) s+f_{02}\left(f_{01}+f_{21}\right)},
$$


so we choose for $\varphi_{1}, \varphi_{2}$, and $\varphi_{3}$

$$
\begin{aligned}
& \varphi_{1}=f_{21}, \\
& \varphi_{2}=f_{21}+f_{02}+f_{01}, \\
& \varphi_{3}=f_{02}\left(f_{21}+f_{01}\right) .
\end{aligned}
$$

This is particularly interesting problem because it illustrates the difference between the solution of the nonlinear and the linearized equations. First note that $f_{21}$ is obviously identifiable. There remain two equations in $f_{02}$ and $f_{21}$. For the nonlinear equations (for $\varphi_{2}$ and $\varphi_{3}$ ), if $f_{02} \neq f_{01}+f_{21}$, there can be onc or two solutions, depending on the relative values of $f_{02}, f_{01}, f_{21}$; if $f_{02}=f_{01}+f_{12}$, there is only one solution. Thus, $f_{01}$ and $f_{02}$ are locally identifiable. For the linearized equations there is only one solution if $f_{02} \neq f_{01}$ $+f_{12}$, but the determinant of the linear equations is zero if $f_{02}=f_{01}+f_{12}$. The latter is an example of a subset of values of locally identifiable parameters for which the parameters appear to be unidentifiable from the analysis of the linearized equations.

Simulation: Same as in Experiment 1a.

The correlation matrix obtained is

$$
\mathbf{C M}=\left[\begin{array}{ccc}
f_{21} & f_{02} & f_{01} \\
1.0 & -0.89 & -0.88 \\
& 1.0 & -1.0 \\
& & 1.0
\end{array}\right]
$$

Note that $r_{23}=-1$; when the parameter values were switched to $f_{01}=2.0$, $f_{02}=0.5, r_{23}$ became -0.78 .

Experiment 1c: Input into 1; Observe 1 and 2.

IR: yes.

OR: yes.

The transfer functions $Q_{1}$ and $Q_{2}$ have been given in Experiments 1a and 1b. Now we have more components than we need:

$$
\begin{aligned}
& \varphi_{1}=f_{21}, \\
& \varphi_{2}=f_{21}+f_{02}+f_{01}, \\
& \varphi_{3}=f_{02}\left(f_{21}+f_{01}\right), \\
& \varphi_{4}=f_{21}+f_{01} .
\end{aligned}
$$

$f_{21}, f_{02}$, and $f_{01}$ are each locally identifiable. For the simulation used for Experiments $1 \mathrm{a}$ and $1 \mathrm{~b}$ we obtain the correlation matrix

$$
\mathbf{C M}=\left[\begin{array}{ccc}
f_{21} & f_{02} & f_{01} \\
& 0.93 & -0.96 \\
& 1.0 & -0.92 \\
& & 1.0
\end{array}\right] .
$$




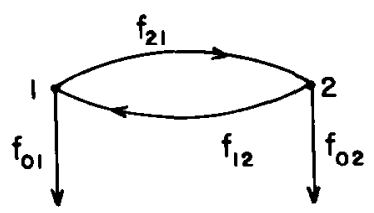

FIG. 2.

Note that now all off-diagonal elements are not \pm 1 , but that the correlations are extremely high, reflecting the nonorthogonality of the fractional transfer coefficients in compartmental kinetics.

B. EXAMPLE 2

For the next example consider the model shown in Figure 2, again for impulsive inputs into compartment 1 .

Experiment 2a: Input into 1, Observe 1.

IR: yes.

OR: yes.

The transfer function for the observed compartment is given by

$$
Q_{1}=\frac{s+f_{02}+f_{12}}{s^{2}+s\left(f_{01}+f_{02}+f_{12}+f_{21}\right)+\left(f_{01} f_{02}+f_{01} f_{12}+f_{02} f_{21}\right)} \text {. }
$$

Thus we have for $\varphi$

$$
\begin{aligned}
& \varphi_{1}=f_{02}+f_{12}, \\
& \varphi_{2}=f_{01}+f_{02}+f_{12}+f_{21}, \\
& \varphi_{3}=f_{01} f_{02}+f_{01} f_{12}+f_{02} f_{21} .
\end{aligned}
$$

None of the parameters are locally identifiable.

Simulation: $f_{21}=1.5, f_{12}=1.25, f_{01}=0.75, f_{02}=2.0 . G(\mathbf{x}, \boldsymbol{\theta}, \mathbf{B u}, t)$ was calculated at 50 points over the interval $[0,1]$. Now $\mathbf{g}^{T} \mathbf{g}$ is of rank 3 .

The correlation matrix obtained is given by

$$
\mathbf{C .}=\left[\begin{array}{rrrr}
f_{21} & f_{12} & f_{01} & f_{02} \\
1.0 & -1 & -1 & 1 \\
& 1 & 1 & -1 \\
& & 1 & -1 \\
& & & 1
\end{array}\right] .
$$

Note that all off-diagonal elements are \pm 1 . 
Experiment 2b: Input into 1, Observe 2.

IR: yes.

OR: yes.

The transfer function for the observed compartment is given by

$$
Q_{2}=\frac{f_{21}}{s^{2}+s\left(f_{01}+f_{02}+f_{12}+f_{21}\right)+\left(f_{01} f_{02}+f_{01} f_{12}+f_{02} f_{21}\right)} .
$$

The components of $\varphi$ are

$$
\begin{aligned}
& \varphi_{1}=f_{21}, \\
& \varphi_{2}=f_{01}+f_{02}+f_{12}+f_{21}, \\
& \varphi_{3}=f_{01} f_{02}+f_{01} f_{12}+f_{02} f_{21} .
\end{aligned}
$$

Now $f_{21}$ is locally identifiable, but the remaining transfer coefficients are not.

For the same simulation used in Experiment $2 \mathrm{a}$, the correlation matrix is now

$$
\mathbf{C M}=\left[\begin{array}{cccc}
f_{21} & f_{12} & f_{01} & f_{02} \\
& -0.04 & -0.05 & -0.05 \\
& 1 & 1 & -1 \\
& & 1 & -1 \\
& & & 1
\end{array}\right]
$$

Note again that the off-diagonal elements for the submatrix corresponding to the non-identifiable transfer coefficients are \pm 1 .

Expcriment 2c: Input 1; Obscrve 1 and 2. Now $\varphi$ has four components - those given in (42) and (45) - and all fractional transfer coefficients are locally identifiable. For the simulation used for Experiments $2 \mathrm{a}$ and $2 \mathrm{~b}$ the correlation matrix now becomes

$$
\mathbf{C M}=\left[\begin{array}{cccr}
1 & 0.48 & -0.65 & 0.61 \\
& 1 & -0.29 & -0.28 \\
& & 1 & -0.95 \\
& & & 1
\end{array}\right]
$$

\section{EXAMPLE 3}

Now consider the catenary system shown in Figure 3. Again we consider experiments with impulsive inputs into 1 . 


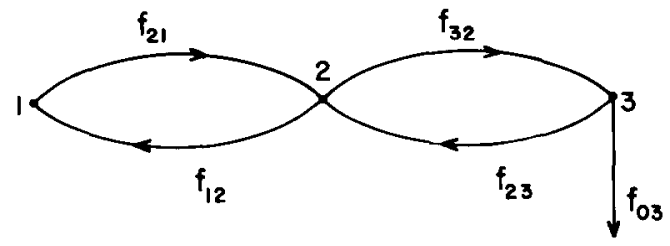

FIG. 3.

Experiment 3a: Input into 1, Observe 1.

IR: yes.

OR: yes.

The transfer function is given by

$$
Q_{1}=\frac{s^{2}+s\left(f_{12}+f_{32}+f_{23}+f_{03}\right)+\left(f_{12} f_{23}+f_{12} f_{03}+f_{03} f_{32}\right)}{\Delta}
$$

where

$$
\begin{aligned}
\Delta= & s^{3}+s^{2}\left(f_{12}+f_{21}+f_{23}+f_{32}+f_{03}\right) \\
& +s\left(f_{12} f_{23}+f_{12} f_{03}+f_{03} f_{32}+f_{21} f_{32}+f_{21} f_{23}+f_{03} f_{21}\right)+f_{03} f_{21} f_{32} .
\end{aligned}
$$

The coefficients give the following components for $\varphi$ :

$$
\begin{aligned}
\varphi_{1} & =f_{12}+f_{32}+f_{23}+f_{03}, \\
\varphi_{2} & =f_{12} f_{23}+f_{12} f_{03}+f_{03} f_{32}, \\
\varphi_{3} & =f_{12}+f_{21}+f_{23}+f_{32}+f_{03}=\varphi_{1}+f_{21}, \\
\varphi_{4} & =f_{12} f_{23}+f_{12} f_{03}+f_{03} f_{32}+f_{21} f_{32}+f_{21} f_{23}+f_{03} f_{21} \\
& =\boldsymbol{\varphi}_{2}+f_{21} f_{32}+f_{21} f_{23}+f_{03} f_{21}, \\
\varphi_{5} & =f_{03} f_{21} f_{32} .
\end{aligned}
$$

For the simulation the following values were used: $f_{21}=2.0, f_{12}=3.0$, $f_{32}=1.5, f_{23}=1.0, f_{03}=2.25 . G^{0}$ was calculated at 50 points over the interval $[0,10]$.

The correlation matrix obtained is given by

$$
\mathbf{C M}=\left[\begin{array}{lllll}
f_{21} & f_{12} & f_{32} & f_{23} & f_{03} \\
& 0.97 & 0.91 & 0.87 & 0.67 \\
& 1 & 0.98 & 0.95 & 0.78 \\
& & 1 & 0.99 & 0.88 \\
& & & 1 & 0.93 \\
& & & & 1
\end{array}\right] .
$$


Note that although all parameters appear to be locally identifiable at $\theta^{\circ}$, one must anticipate difficulties in estimating the parameters because of the high correlations between them.

Experiment 3b: Input into 1; Observe 2. Now the transfer function for the experiment is

$$
Q_{2}=\frac{f_{21} s+f_{21}\left(f_{23}+f_{03}\right)}{\Delta}
$$

The components of $\varphi$ are

$$
\begin{aligned}
& \varphi_{1}=f_{21}, \\
& \varphi_{2}=f_{21} f_{23}+f_{21} f_{03} \text {, } \\
& \left.\begin{array}{l}
\varphi_{3} \\
\varphi_{4} \\
\varphi_{5}
\end{array}\right\} \text { same as in (49). }
\end{aligned}
$$

All parameters are locally identifiable if $f_{21}-f_{03}-f_{23} \neq 0$.

For the same simulation used for Experiment $3 a$ the correlation matrix is

$$
\mathbf{C M}=\left[\begin{array}{ccccc}
f_{21} & f_{12} & f_{32} & f_{23} & f_{03} \\
1 & 0.62 & -0.05 & 0.92 & 0.63 \\
& 1 & -0.80 & 0.71 & 0.99 \\
& & 1 & -0.15 & -0.76 \\
& & & 1 & 0.75 \\
& & & & 1
\end{array}\right] .
$$

Again all parameters are identifiable, but estimation of the parameters should be much easier than in Experiment 3a. If one has the choice, Experiment $3 b$ is better for estimating most of the parametcrs.

Experiment 3c: Input into 1, Observe 3.

IR: yes.

OR: yes.

The transfer function for the experiment is

$$
Q_{3}=\frac{f_{21} f_{32}}{\Delta}
$$

Now there are only four components to $\varphi$ :

$$
\begin{aligned}
& \varphi_{1}=f_{21} f_{32}, \\
& \varphi_{2}, \varphi_{3}, \varphi_{4} \text { are the same as } \varphi_{3}, \varphi_{4}, \varphi_{5} \text { of (49). }
\end{aligned}
$$


Thus, all parameters cannot be identifiable; $f_{03}$ is identifiable from $\varphi_{1}$ and $\varphi_{4}$.

The correlation matrix obtained was

$$
\mathbf{C M}=\left[\begin{array}{ccccc}
f_{21} & f_{12} & f_{32} & f_{23} & f_{03} \\
1 & 0.93 & -1.0 & -1.0 & -0.20 \\
& 1 & -0.93 & -0.95 & -0.16 \\
& & 1 & 1.0 & 0.20 \\
& & & 1 & 0.19 \\
& & & & 1
\end{array}\right] .
$$

Note that $f_{21}, f_{32}, f_{23}$ clearly fall into set (ii). But $f_{12}$ appears to be locally unidentifiable, and we are not certain yet whether not finding +1 or -1 for $r_{12}, r_{23}$, and $r_{24}$ is a result of numerical problems in the calculation or results from an incomplete analysis of all possibilities for members of set (ii).

Experiments $3 d$ and $3 e$. For an experiment in which compartments 1 and 3 are observed, the correlation matrix is

$$
\mathbf{C M}=\left[\begin{array}{ccccc}
f_{21} & f_{12} & f_{32} & f_{23} & f_{03} \\
1.0 & 0.91 & -0.12 & -0.28 & 0.03 \\
& 1.0 & 0.22 & -0.01 & 0.02 \\
& & 1 & 0.96 & -0.05 \\
& & & 1.0 & -0.01 \\
& & & & 1.0
\end{array}\right] .
$$

Note that the correlations have decreased markedly but the interactions between $f_{21}$ and $f_{21}$ and between $f_{23}$ and $f_{32}$ remain high.

For observation of compartments 2 and 3 the correlation matrix becomes

$$
\mathbf{C M}=\left[\begin{array}{ccccc}
f_{21} & f_{12} & f_{32} & f_{23} & f_{03} \\
1.0 & 0.92 & -0.04 & -0.02 & -0.15 \\
& 1.0 & -0.28 & -0.26 & -0.20 \\
& & 1 & 0.99 & 0.00 \\
& & & 1.0 & 0.04 \\
& & & & 1
\end{array}\right] .
$$

Note that $r_{35}=0.00$ is not a zero correlation that indicates a parameter is missing from the observations; it is a very small correlation rounded to 0.00 and indicates practically zero pairwise correlation between $f_{32}$ and $f_{03}$.

\section{OPTIMAL SAMPLING DESIGN AND LOCAL IDENTIFIABILITY}

The term "design of experiments" is used for a broad class of problems involving optimization of various facets of the experiments, including the choice of inputs and observations, number of samples, and sampling times. 
Once an experiment is chosen, extraneous constraints such as costs of the experiment and biological limitations on what can be done pretty well put bounds on the maximum sampling time and on number of samples that can be taken. What remains is to optimize the times at which the samples are taken so as to make parameter estimation most efficient. In brief the optimal sampling problem can be formulated as follows: given

(i) an initial estimate for the parameter values,

(ii) that sampling is restricted to the interval in time $0<t \leqslant T$,

(iii) that the number of samples to be taken is $n$,

(iv) the variance of the sampling errors (sometimes information on distributions is available),

find the distribution of sampling times in $(0, T]$ that optimizes the expected value of some function of the variances of the parameter estimates. The choice of criterion function depends on the error structure as well as on the goals of the experiment.

\section{A. THE CLASSICAL APPROACH BY LEAST SQUARES}

Again we start with the linearized form of the observations, but with additive error $\epsilon_{i}$, a random variable of known variance $\sigma_{i}^{2}$, as in Equation (16). However, now weighted least squares must be used

$$
S=\sum_{i=1}^{n} w_{i}\left[\eta_{i}-G_{i}^{0}-\sum_{j} \frac{\partial G_{i}^{0}}{\partial \theta_{j}} \Delta \theta_{j}\right]^{2}
$$

with $w_{i}=1 / \sigma_{i}^{2}$. The solution for $\overline{\Delta \theta}$ is now given by

$$
\left(\mathbf{g}^{T} \mathbf{W g}\right) \widehat{\Delta \boldsymbol{\theta}}=\mathbf{g}^{T} \mathbf{W z}, \quad \mathbf{W}=\operatorname{diag}\left(w_{1}, w_{2}, \ldots, w_{n}\right) .
$$

If the model is locally identifiable, then $\mathbf{g}^{T} \mathbf{W g}$ has an inverse and $\overline{\Delta \theta}$ is given by

$$
\widehat{\Delta \theta}=\left(\mathbf{g}^{T} \mathbf{W g}\right)^{-1} \mathbf{g}^{T} \mathbf{W} \mathbf{z}
$$

Here $\left(\mathbf{g}^{T} \mathbf{W g}\right)^{-1}$ is an approximation to the covariance matrix of $\widehat{\Delta \theta}$. The idea in optimal sampling theory is to choose the sampling times $t_{1}, \ldots, t_{n}$ in $(0, T]$ so as to minimize some measure of the variance of the estimates.

If the model is locally identifiable, a number of criteria for choosing $t_{1}, \ldots, t_{n}$ have been proposed. Box and Lucas [36] pointed out that $\left|\left(\mathbf{g}^{T} \mathbf{W g}\right)^{-1}\right|$ is proportional to the volume of an ellipsoid of constant $\Delta S$ about the estimate and suggested minimizing $\left|\left(\mathbf{g}^{T} \mathbf{W g}\right)^{-1}\right|$. A design derived from this criterion is called a $D$-optimal design. Minimizing the maximum eigenvalue 
of $\left(\mathbf{g}^{T} \mathbf{W} \mathbf{g}\right)^{-1}$ is called an $E$-optimal design. For the maximum likelihood approach, minimizing a functional of the covariance matrix of the estimates means maximizing the same functional of Fisher's information matrix. The designs obtained from quite a few criteria have been studied by now; for a discussion of the properties of these designs see Landaw [37] and Fedorov [38].

\section{B. AN APPROACH BY WAY OF SENSITIVITY OF LEAST SQUARES}

The above results are well known, but they provide little insight and no guidance on what to do if the concern is to obtain optimal estimates of a few locally identifiable parameters in a model that is not locally identifiable. A more general approach, which is very instructive, is to note that in general we want to maximize the sensitivity of $S$ to change in each of the parameters to be estimated. The derivation is easy and generally more instructive for the practitioner; it starts with the sum of squares without linearizing $G$ :

$$
S=\sum_{i=1}^{n} w_{i}\left[\eta_{i}-G\left(\mathbf{x}_{i}, \boldsymbol{\varphi}, \mathbf{B} \mathbf{u}, t_{i}\right)\right]^{2}
$$

Now expand $S$ :

$$
S=S^{0}+\sum_{j} \frac{\partial S^{0}}{\partial \theta_{j}} \Delta \theta_{j}+\sum_{j} \sum_{k} \frac{1}{2} \frac{\partial^{2} S^{0}}{\partial \theta_{j} \partial \theta_{k}} \Delta \theta_{j} \Delta \theta_{k}
$$

As before the superscript ${ }^{0}$ indicates that the term is evaluated at $\theta=\theta^{0}$. The terms in (63) are given by

$$
\begin{aligned}
S^{0} & =\sum w_{i}\left[\eta_{i}-G_{i}^{0}\right]^{2}, \\
\frac{\partial S^{0}}{\partial \theta_{j}} & =0=-2 \sum_{i} w_{i}\left[\eta_{i}-G_{i}^{0}\right] \frac{\partial G_{i}^{0}}{\partial \theta_{j}} \\
\frac{\partial^{2} S^{0}}{\partial \theta_{j} \partial \theta_{k}} & =-2 \sum_{i} w_{i}\left[\eta_{i}-G_{i}^{0}\right] \frac{\partial^{2} G_{i}^{0}}{\partial \theta_{j} \partial \theta_{k}}+2 \sum_{i} w_{i} \frac{\partial G_{i}^{0}}{\partial \theta_{j}} \frac{\partial G_{i}^{0}}{\partial \theta_{k}} .
\end{aligned}
$$

Note that $\partial S^{0} / \partial \theta_{j}$ must be zero at the minimum in $S$. With (64)-(66), (63) may be rewritten as

$$
S-S^{0}=\sum_{i} \sum_{j} \sum_{k}\left[-w_{i}\left(\eta_{i}-G_{i}^{0}\right) \frac{\partial^{2} G_{i}^{0}}{\partial \theta_{j} \partial \theta_{k}}+w_{i} \frac{\partial G_{i}^{0}}{\partial \theta_{j}} \frac{\partial G_{i}^{0}}{\partial \theta_{k}}\right] \Delta \theta_{j} \Delta \theta_{k}
$$


But $E\left[\eta_{i}-G_{i}^{0}\right]=0$, so we neglect the first term in (67) to obtain

$$
\begin{aligned}
S-S^{0} & =\sum_{j} \sum_{k}\left[\sum_{i} w_{i} \frac{\partial G_{i}^{0}}{\partial \theta_{j}} \frac{\partial G_{i}^{0}}{\partial \theta_{k}}\right] \Delta \theta_{j} \Delta \theta_{k} \\
& =\Delta \boldsymbol{\theta}^{T} \mathbf{g}^{T} W \mathbf{g} \Delta \boldsymbol{\theta}
\end{aligned}
$$

For given $\Delta \theta_{j}, \Delta \theta_{k}$ we want to pick the samples so as to maximize their coefficients, i.e. the proper elements of $g^{T} \mathbf{W g}$; that choice maximizes the sensitivity of $S$ to change in $\theta_{j}$ and $\theta_{k}$ around the minimum $S^{0}$. So either approach leads us to look at the effect of choice of $t_{1}, \ldots, t_{n}$ on the elements of $\mathbf{g}^{T} \mathbf{W g}$. But with this approach, it is clear we need not concern ourselves with whether or not $\mathbf{g}^{T} \mathbf{W g}$ has an inverse; simply look at the appropriate components of $\mathbf{g}^{r} \mathbf{W g}$.

It should be obvious by now that if we have initial estimates of the parameters, then local identifiability, estimability, and optimal sampling design involve rather similar considerations, with the difference that for local identifiability and estimability no error is involved, but the $\sigma_{i}^{2}$ for the measurements must be taken into account for optimal sampling. Furthermore, both are then inherently iterative processes, and we can expect rapid improvement in design as soon as the estimates become based on data. For applications, we foresee writing software for optimal sampling design in which the check of local identifiability is built in as a first step.

\section{DISCUSSION}

To recapitulate, for practical application to the design of experiments we emphasize the following:

(1) An important distinction is made between structural, experimental, and observational parameters.

(2) It is important to focus on the parameters significant for the problem at hand, so we need to develop practical approaches to examining local identifiability for particular parameters, whether or not the model is locally identifiable.

(3) It is important to look at local identifiability and estimability at specific values of parameters, the initial values. In some problems it may be necessary to examine these for a number of values for the parameters.

(4) Local identifiability based on linearization around initial estimates has led us to examine the information contained in the a priori correlation matrix. By "a priori" correlation matrix we mean that calculated in the identifiability stage from generated data without addition of error. We have proposed the term estimability for the a priori problem of determining interdependence of locally identifiable parameters, and suggest the use of the a priori correlation coefficient to measure estimability. 
(5) We want to distinguish three classes of parameters: those that are locally identifiable, those that are observable (i.e. appear in the observations, but are not locally identifiable), and those that do not appear in the observations. The a priori correlation matrix provides information on these three classes.

(6) The approach to identifiability and estimability at initial estimates and by way of least squares on a finite sample generated for the initial estimates fits directly into the approach to optimal sampling theory, so the latter is then a direct extension from the identifiability study.

In this study we have been led to look at the a priori correlation matrix, and we emphasize the need for more work on it. It clearly separates the parameters that do not appear in the observations from those that do, and also gives information on local identifiability and on estimability of the locally identifiable parameters at the initial estimates. We are not sure we have found all of the possible patterns of correlations betwecn parameters in set (ii), but since poor estimability is in practice almost as big a problem as local unidentifiability, the occurrence of either suggests one should search for other experimental designs. If a parameter is not identifiable, it is not estimable; but a parameter may be identifiable and still be poorly estimable. Brown and Godfrey [39] used the term determinacy for a broader idea that included estimability; determinacy included experimental design conditions and effects of measurement error. We also point out two approaches to estimating parameters that may not be locally identifiable. One $[9,40]$ is to use other information to fix enough parameters to make those that are of interest identifiable; the estimates obtained are then conditional estimates. The other is interval identifiability or quasiidentifiability, due to DiStefano [41]; the idea here is to try to restrict parameters to intervals small enough so that they are "identifiable for practical purposes."

The development summarized above focuses on one model of a system and a given experiment. It should be pointed out that the method given can also be used to compare the values of different experiments on one model and thus is useful for choosing the best experiments. A natural extension is to the comparison of the predictions of a number of models for a series of experiments. All of these are important for the design of experiments in the broad sense and for distinguishing between models.

Finally it is important to emphasize that the identifiability problem refers to identifiability of the parameters of a model, and the optimal sampling design obtained is for the model in question, so both can be done on the model without reference to the real system. But the applicability of the results depends on whether the model is indeed a good model of the system. The difference between results obtained on the real system and a model includes model bias, or model misspecification error, and is important for 
testing models. We have not examined those issues here, but want to emphasize their importance and point to some references on that important problem $[7,42]$.

We thank Drs. John Hearon and Eric Walter for their incisive reviews of two prior versions of this paper.

\section{REFERENCES}

1 T. C. Koopmans and O. Reiersol, Identification of structural characteristics, $A n n$. Math. Statist. 21:165-181 (1950).

2 F. M. Fisher, Generalization of the rank and order conditions for identifiability, Econometrica 27:431-477 (1959).

3 K. J. Astrom and P. Cykhoff, System identification-a survey, Automatica 7:123-162 (1971).

4 R. K. Mehra, Topics in stochastic control theory-identification in control and econometrics: Similarities and differences, Ann. Econ. Soc. Meas. 3:24-47 (1974).

5 R. Bellman and K. J. Astrom, On structural identifiability, Math. Biosci. 7:329-339 (1970).

6 V. V. Nguyen and E. F. Wood, Review and unification of linear identifiability concepts, SIAM Rev. 24:34-51 (1982).

7 E. Walter, Identifiability of State Space Models, Lecture Notes in Biomathematics No. 46, Springer, Berlin, 1982.

8 D. H. Anderson, Compartmental Modeling and Tracer Kinetics, Lecture Notes in Biomathematics No. 50, Springer, Berlin, 1983.

9 J. A. Jacquez, The inverse problem for compartmental systems, Math. Comput. Simulation 24:452-459 (1982).

$10 \mathrm{~K}$. Glover and J. C. Willems, Parametrizations of linear dynamical systems: canonical forms and identifiability, IEEE Trans. Automat. Control 19:640-646 (1974).

$11 \mathrm{~K}$. Glover and J. C. Willems, On the identifiability of linear dynamical systems, in Proceedings of the 3rd IFAC Symposium on Identification and System Parameter Estimation, The Hague, 1973, pp. 867-870.

$12 \mathrm{M}$. S. Grewal and K. Glover, Identifiability of linear and nonlinear dynamical systems, IEEE Trans. Automat. Control 21:833-837 (1976).

13 C. Cobelli and J. J. DiStefano III, Parameter and structural identifiability concepts and ambiguities: A critical review and analysis, Amer. J. Physiol. 239:R7-R24 (1980).

14 C. Cobelli, A. Lepschy, and G. Romanin-Jacur, Identifiability of compartmental systems and related structural properties, Math. Biosci. 44:1-18 (1979).

15 J. A. Jacquez, Compartmental models of biological systems: Lincar and nonlinear, in Applied Nonlinear Analysis (V. Lakshmikantham, Ed.), Academic, New York, 1979, pp. 185-205.

16 C. Cobelli, A. Lepschy, and G. Romanin-Jacur, Identifiability results on some constrained compartmental systems, Math. Biosci. 47:173-195 (1979).

17 J. Delforge, New results on the problem of identifiability of a linear system, Math. Biosci. 52:73-96 (1980).

18 J. Delforge, Necessary and sufficient conditions for local identifiability of a system with linear compartments, Math. Biosci. 54:159-180 (1981).

19 J. J. DiStefano III, Complete parameter bounds and quasidentifiable linear systems, Math. Biosci. 65:51-68 (1983). 
$20 \mathrm{~J}$. Eisenfeld, On identifiability of impulse-response in compartmental systems, Math. Biosci. 47:15-34 (1979).

21 M. Milanese and G. P. Molino, Structural identifiability of compartmental models and pathophysiological information from the kinetics of drugs, Math. Biosci. 26:175-190 (1975).

22 J. P. Norton, Normal-mode identifiability analysis of linear compartmental systems in linear stages, Math. Biasci. 50:95-115 (1980).

23 J. P. Norton, An investigation of the sources of nonuniqueness in deterministic identifiability, Math. Biosci. 60:89-108 (1982).

24 C. Travis and G. Haddock, On structural identification, Math. Biosci. 56:157-173 (1981).

25 H. Pohjanpalo, System identifiability based on the power series expansion of the solution, Math. Biosci. 4:21-33 (1978).

26 S. Vajda, Structural equivalence of linear systems and compartmental models, Math. Biosci. 55:39-64 (1981).

27 E. Walter and Y. Lecourtier, Unidentifiable compartmental models. What to do? Math. Biosci. 56:1-25 (1981).

28 I. Delforge, Sur l'identifiabilité et l'identification des modèles linéaires, Thèse d'Etat, Univ. d'Angers, 1984.

29 C. T. Chen, Introduction to Linear System Theory, Holt, Rinehart and Winston, New York, 1970.

30 A. Raksanyi, Y. Lecourtier, E. Walter, and A. Venot, Identifiability and distinguishability testing via computer algebra, Math. Biosci, 77:245-266 (1985).

31 M. Berman and R. Schoenfeld, Invariants in experimental data on linear kinetics and the formulation of models, J. Appl. Phys. 27:1361-70 (1956).

32 J. A. Jacquez, Compartmental Analysis in Biology and Medicine, 2nd ed., Univ. of Michigan Press, 1985, Chapter 14.

33 A. C. Aitken, Determinants and Matrices, Oliver and Boyd, Edinburgh, 1962.

34 M. Milanese and N. Sorrentino, Decomposition methods for the identifiability analysis of large systems, Internat. J. Control 28:71-79 (1978).

35 R. E. Kalman, Mathematical description of linear dynamical systems, SIAM J. Control 1:152-192 (1963).

36 G. E. R. Box and H. L. Lucas, Design of experiments in non-linear situations, Biometrika 46:77-90 (1959).

37 E. M. Landaw, Optimal experimental design for biologic compartmental systems with applications to pharmacokinetics, Ph.D. Thesis, Univ. of California, Los Angeles, 1980.

38 V. V. Fedorov, Theory of Optimal Experiments, Academic, New York, 1972.

39 R. F. Brown and K. R. Godfrey, Problems of determinacy in compartmental modeling with application to bilirubin kinetics, Math Biosci. 40:205-224 (1978).

40 J. A. Jacquez, Compartmental Analysis in Biology and Medicine, 1st ed., Elsevier. Amsterdam, 1972, Chapter 7.

41 J. J. DiStefano, III, Complete parameter bounds and quasiidentifiable linear systems, Math. Biosci. 65:51-658 (1983).

42 F. Y, Hadaegh and G. A. Bekey, Near-identifiability of dynamical systems, Math Biosci. 77:325-340 (1985). 